OPEN ACCESS

Edited by:

Tomas Vaisar,

University of Washington,

United States

Reviewed by:

Noemie Clouet-Foraison, University of Washington,

United States

Hiroshi Yoshida,

Jikei University Kashiwa

Hospital, Japan

*Correspondence:

Shiqun Chen

shiqunchen@126.com

Liling Chen

chenliling19790206@163.com

Jin Liu

ljaw397017568@163.com

†These authors have contributed equally to this work

Specialty section:

This article was submitted to Lipids in Cardiovascular Disease,

a section of the journal

Frontiers in Cardiovascular Medicine

Received: 25 July 2021 Accepted: 15 October 2021 Published: 17 November 2021

Citation:

Huang Z, Yang Y, Lu J, Liang J, He Y,

Yu Y, Huang $H, L i Q$, Wang $B, L i S$, Yan Z, Xu D, Liu Y, Chen K, Huang Z, $\mathrm{Ni}$ J, Liu J, Chen L and Chen S (2021)

Association of

Lipoprotein(a)-Associated Mortality and the Estimated Glomerular

Filtration Rate Level in Patients Undergoing Coronary Angiography: A 51,500 Cohort Study.

Front. Cardiovasc. Med. 8:747120. doi: 10.3389/fcvm.2021.747120

\section{Association of} Lipoprotein(a)-Associated Mortality and the Estimated Glomerular Filtration Rate Level in Patients Undergoing Coronary Angiography: A 51,500 Cohort Study

\begin{abstract}
Zhidong Huang ${ }^{1 \dagger}$, Yanfang Yang ${ }^{2,3+}$, Jin $\mathrm{Lu}^{2,3+}$, Jingjing Liang ${ }^{4}$, Yibo $\mathrm{He}^{1}$, Yaren $\mathrm{Yu}^{5}$, Haozhang Huang ${ }^{1}$, Qiang $\mathrm{Li}^{1}$, Bo Wang ${ }^{1}$, Shanggang $\mathrm{Li}^{6}$, Zelin Yan ${ }^{4}$, Danyuan $\mathrm{Xu}^{1}$, Yong Liu ${ }^{1}$, Kaihong Chen ${ }^{3}$, Zhigang Huang ${ }^{6}$, Jindong $\mathrm{Ni}^{6}$, Jin Liu ${ }^{1 *}$, Liling Chen ${ }^{3 *}$ and Shiqun Chen ${ }^{1 *}$

${ }^{1}$ Department of Cardiology, Guangdong Provincial Key Laboratory of Coronary Heart Disease Prevention, Guangdong Cardiovascular Institute, Guangdong Provincial People's Hospital, Guangdong Academy of Medical Sciences, Guangzhou, China, ${ }^{2}$ The Graduate School of Clinical Medicine, Fujian Medical University, Fuzhou, China, ${ }^{3}$ Department of Cardiology, Longyan First Affiliated Hospital of Fujian Medical University, Longyan, China, ${ }^{4}$ The Second School of Clinical Medicine, Southern Medical University, Guangzhou, China, ${ }^{5}$ Department of Cardiology, The First People's Hospital of Foshan, Foshan, China, ${ }^{6}$ Department of Public Health, Guangdong Medical University, Dongguan, China
\end{abstract}

Background: High lipoprotein(a) is associated with poor prognosis in patients at high risk for cardiovascular disease. Renal function based on the estimated glomerular filtration rate (eGFR) is a potential risk factor for the change of lipoprotein(a). However, the regulatory effect of eGFR stratification on lipoprotein(a)-associated mortality has not been adequately addressed.

Methods: 51,500 patients who underwent coronary angiography (CAG) or percutaneous coronary intervention $(\mathrm{PCl})$ were included from the Cardiorenal ImprovemeNt (CIN) study (ClinicalTrials.gov NCT04407936). These patients were grouped according to lipoprotein(a) quartiles (Q1-Q4) stratified by eGFR categories $(<60$ and $\geq 60 \mathrm{~mL} / \mathrm{min} / 1.73 \mathrm{~m}^{2}$ ). Cox regression models were used to estimate hazard ratios $(\mathrm{HR})$ for mortality across combined eGFR and lipoprotein(a) categories.

Results: The mean age of the study population was $62.3 \pm 10.6$ years, $31.3 \%$ were female $(n=16,112)$. During a median follow-up of 5.0 years (interquartile range: $3.0-7.6$ years), $13.0 \%$ ( $n=6,695)$ of patients died. Compared with lipoprotein(a) Q1, lipoprotein(a) Q2-Q4 was associated with 10\% increased adjusted risk of death in all patients (HR: 1.10 [95\% Cl: 1.03-1.17]), and was strongly associated with about $23 \%$ increased adjusted risk of death in patients with eGFR $<60 \mathrm{~mL} / \mathrm{min} / 1.73 \mathrm{~m}^{2}$ (HR: 1.23 [95\% Cl: 1.08-1.39]), while such association was not significant in patients with eGFR $\geq 60 \mathrm{~mL} / \mathrm{min} / 1.73 \mathrm{~m}^{2}$ (HR: 1.05 [95\% Cl: 0.97-1.13]). P for interaction between lipoprotein(a) (Q1 vs. Q2-Q4) and eGFR ( $\geq 60$ vs. eGFR $<60 \mathrm{~mL} / \mathrm{min} / 1.73 \mathrm{~m}^{2}$ ) on all-cause mortality was 0.019 . 
Conclusions: Elevated lipoprotein(a) was associated with increased risk of all-cause mortality and such an association was modified by the baseline eGFR in CAG patients. More attention should be paid to the patients with reduced eGFR and elevated lipoprotein(a), and the appropriate lipoprotein(a) intervention is required.

Keywords: lipoprotein(a), estimated glomerular filtration rate, all-cause mortality, coronary angiography, renal function

\section{INTRODUCTION}

Lipoprotein(a) is a low-density lipoprotein (LDL) particle covalently bound to a large glycoprotein, apolipoprotein(a) [apo(a)] (1). Recent advances have once again thrust lipoprotein(a) into the clinical spotlight that large evidence in genetic and epidemiology verified that high lipoprotein(a) level was an independent risk factor for morbidity and mortality of atherosclerotic cardiovascular disease (2-6). Many pathogenic pathways are activated in this complex process including proatherogenic, pro-thrombotic, and pro-oxidative properties (4). Lipoprotein(a) has been recommended to identify high risk patients with coronary artery disease $(C A D)$ by the last European and American cholesterol management guidelines $(7,8)$. Several potent and specific therapies including proprotein convertase subtilisin/kexin type 9 (PCSK9) inhibitors and antisense oligonucleotides, are currently being developed clinically to lower plasma lipoprotein(a) concentrations $(9,10)$.

The metabolism of lipoprotein(a), though still not wellunderstood, appears to be related to renal function. The reason for increased lipoprotein(a) concentrations in renal dysfunction patients probably derives from the decreased clearance or an increased synthesis in the liver caused by the proteinuria $(11,12)$. Understanding the potential interplay of lipoprotein(a) and renal function may allow optimal personalized treatment of patients with or without multiple risk factors. To date, however, limited data exist on the association between lipoprotein(a) and the prognosis in patients with different degrees of renal dysfunction.

Given that level of the estimated glomerular filtration rate (eGFR) could reflect the degree of renal insufficiency (13, 14). Our objective was to examine the relationship between lipoprotein(a) and all-cause mortality across categories of eGFR.

\section{METHOD}

\section{Data Sources and Study Population}

The Cardiorenal ImprovemeNt (CIN) study is a single-center, retrospective and observational cohort study, enrolling 88,938 consecutive patients undergoing coronary angiography (CAG) or percutaneous coronary intervention (PCI) in Guangdong Provincial People's Hospital, Guangdong, China, hospitalized in between January 2007 to December 2018 (ClinicalTrials.gov NCT04407936). PCI was performed following standard clinical practice guidelines. Exclusive criteria included: (a) patients without data of baseline lipoprotein(a) $(n=13,848)$; (b) patients without data of baseline eGFR $(n=15,165)$; c) and patients without follow-up data $(n=8,425)$. These data were missing randomly. Eventually, 51,500 patients were included (Supplementary Figure 1).

\section{Baseline Data Collection}

From January 2007 to December 2018, data were extracted from the electronic clinical management records system of the Guangdong Provincial People's Hospital. We had access to all primary and secondary care records. The baseline information included demographic characteristics, coexisting conditions, laboratory examinations, and medications at discharge. Blood samples except lipid profiles were collected at admission or before CAG and PCI. The lipoprotein(a) was measured by an overnight fasting venous blood sample. The death of patients after discharge were recorded by the attending physician or a trained research assistant at the follow-up.

\section{Measurement of Lipoprotein(a)}

Lipoprotein(a) mass was measured by the latex-enhanced immunoturbidimetric assay using an automatic biochemical analyzer (Beckman AU5800, USA). The detection principles were as follows: Denka antibodies which were anti-lipoprotein(a) polyclonal antibodies coupled to latex microparticles react with lipoprotein(a) in the sample to form an antigen/antibody complex leading to agglutination causing turbidity of the reaction mixture. The absorbance of the compound was proportional to the concentration of lipoprotein(a) in the specimen (manufacturer: Beckman Coulter, Brea, California, unit: $\mathrm{mg} / \mathrm{dL}$, normal range: $<30 \mathrm{mg} / \mathrm{dL}$ ). Measurements are linear in the range of $5.0-80.0 \mathrm{mg} / \mathrm{dL}$. The intraassay coefficient of variation was $\leq 10 \%$. Danka calibrator samples were used for correction to minimize the impact of apo(a) isoforms.

\section{Endpoint and Clinical Definition}

The primary endpoint was all-cause mortality which was monitored and recorded by trained nurses and research assistants through outpatient interviews and telephones. The level of lipoprotein(a) was quartered into four groups (quartiles Q1:0-8.30 $\mathrm{mg} / \mathrm{dL}, \quad$ Q2:8.30-15.30 $\mathrm{mg} / \mathrm{dL}, \quad$ Q3:15.30-32.10 $\mathrm{mg} / \mathrm{dL}, \mathrm{Q} 4: 32.10-522.44 \mathrm{mg} / \mathrm{dL})$. The eGFR was calculated by the Modification of Diet in Renal Disease formula (15), and was divided into 2 categories of eGFR $(<60$, $\left.\geq 60 \mathrm{~mL} / \mathrm{min} / 1.73 \mathrm{~m}^{2}\right)$. eGFR $\left[\mathrm{mL} /\left(\mathrm{min} \cdot 1.73 \mathrm{~m}^{2}\right)\right]=186 \times$ $[\mathrm{SCr}(\mu \mathrm{mol} / \mathrm{L}) \times 0.011312]^{-1.154} \times$ age $^{-0.203}($ if female $\times 0.762)$ (16). Anemia was defined as a hematocrit $\leq 39 \%$ (male) or $\leq 36 \%$ (female). Congestive heart failure ( $\mathrm{CHF}$ ) was defined as New York Heart Association class $>2$ or Killip class $>1$ (17). $\mathrm{CAD}$, acute myocardial infarction (AMI), valvular heart disease 
TABLE 1 | Baseline characteristics across eGFR and lipoprotein(a) categories.

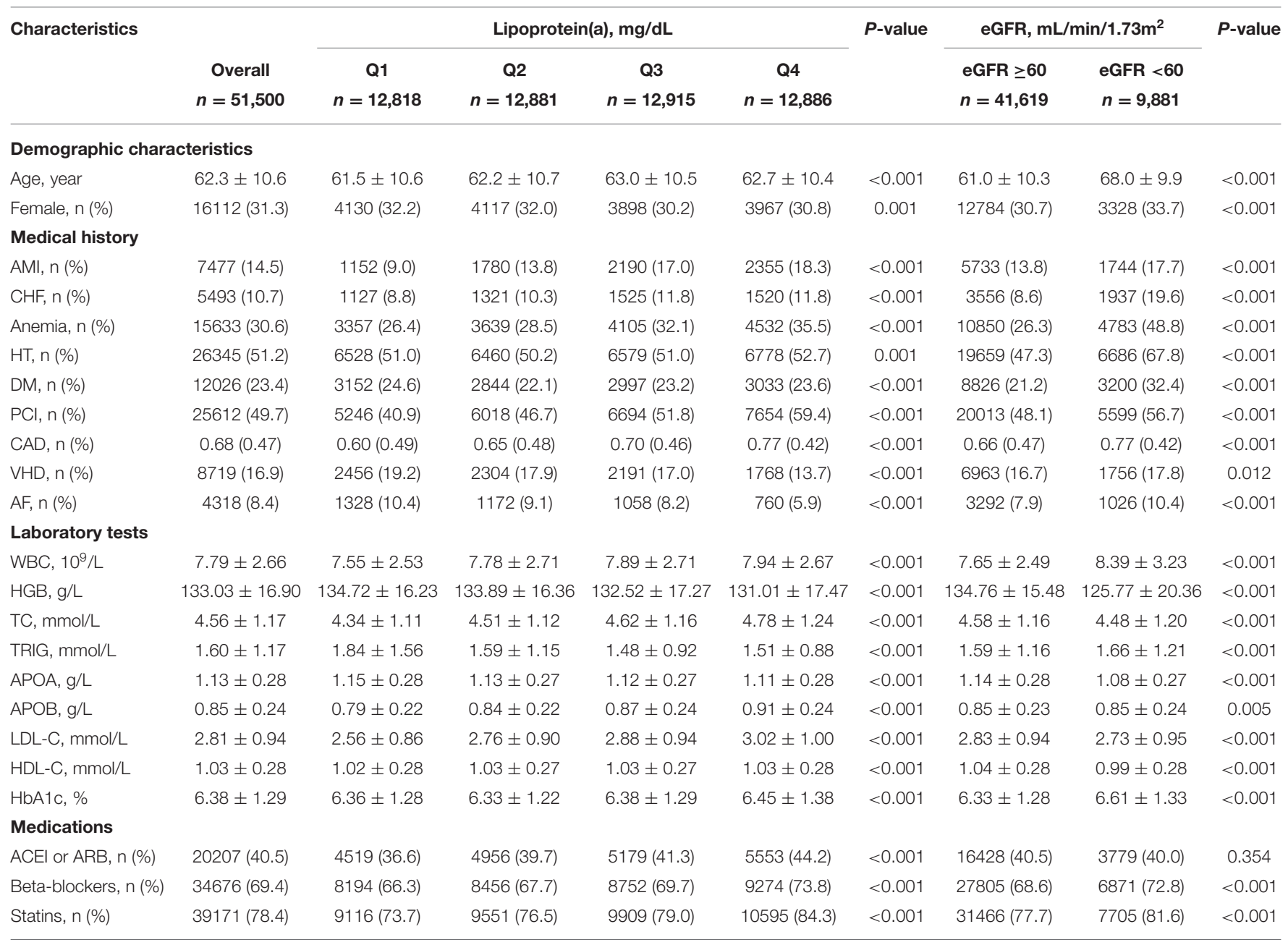

Values are, $n(\%)$ or mean $\pm S D$.

$A M I$, acute myocardial infarction; $C H F$, congestive heart failure; HT, hypertension; DM, diabetes mellitus; PCl, percutaneous coronary intervention; CAD, coronary artery disease; VHD, valvular heart disease; AF, atrial fibrillation; WBC, white blood cell; HGB, hemoglobin; TC, serum total cholesterol; TRIG, triglycerides; APOA, apolipoprotein A; APOB, apolipoprotein

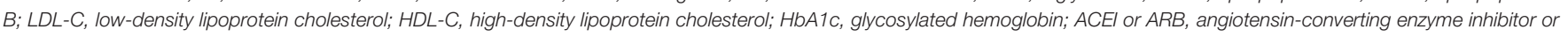
angiotensin receptor blocker.

(VHD), atrial fibrillation (AF), hypertension (HT), and diabetes mellitus (DM) were defined using ICD-10 codes.

\section{Statistical Analysis}

Baseline characteristics are presented as mean \pm SD for continuous variables, and proportions for categorical variables. The differences of baseline characteristics between groups were compared using Student $t$-test for continuous variables and chi-square tests for categorical variables. The differences of characteristics in box plot were compared using Kruskal-Wallis. Time-to-event data among groups are presented graphically using Kaplan-Meier curves and compared by the log-rank test. Multivariable Cox regression models were used to estimate hazard ratios for mortality across combined eGFR and lipoprotein(a) categories, or respectively, with adjustment for major covariables including age, gender, PCI, AMI, HT, DM, anemia, stroke, CHF, CAD, VHD, AF, LDL cholesterol (LDLC), high-density lipoprotein cholesterol (HDL-C), triglycerides (TRIG), angiotensin-converting enzyme inhibitor or angiotensin receptor blocker (ACEI or ARB), beta-blockers; statins. The $p$-value for interactions between categories of eGFR and lipoprotein(a) in all-cause mortality were estimated using the Wald chi-square test. Presented tests were 2-tailed for all, and a $p$ value $<0.05$ was considered statistically significant. All statistical analyses were performed using $R$ (ver. 4.0.3).

\section{RESULT}

There were 51,500 patients included in total in this study. The mean age of the study population was $62.3 \pm 10.6$ years, $31.3 \%$ were female. There were significant differences in prevalence of anemia, AMI, CHF, PCI, CAD and the concentration of serum total cholesterol and LDL-C, and use of statins and ACEI 
TABLE 2 | Lipoprotein(a) and eGFR in relation to all-cause mortality, respectively.

\begin{tabular}{|c|c|c|c|c|c|}
\hline \multirow[t]{2}{*}{ Categories } & \multirow[t]{2}{*}{ Events (\%) } & \multicolumn{2}{|c|}{ Crude } & \multicolumn{2}{|c|}{ Adjusted } \\
\hline & & HR (95\% Cl) & $P$-value & HR $(95 \% \mathrm{Cl})$ & $P$-value \\
\hline \multicolumn{6}{|l|}{ Lipoprotein(a), mg/dL } \\
\hline Continuous variable per 10 units & & $1.002(1.001-1.003)$ & $<0.001$ & $1.001(1.000-1.002)$ & 0.010 \\
\hline \multicolumn{6}{|l|}{ Quartile } \\
\hline Q1 & $1429(11.15 \%)$ & ref & & ref & \\
\hline Q2 & 1661 (12.89\%) & $1.11(1.04-1.20)$ & 0.003 & $1.08(1.00-1.16)$ & 0.043 \\
\hline Q3 & $1813(14.04 \%)$ & $1.21(1.12-1.29)$ & $<0.001$ & $1.10(1.02-1.19)$ & 0.008 \\
\hline Q4 & 1839 (14.27\%) & $1.21(1.13-1.30)$ & $<0.001$ & $1.11(1.03-1.19)$ & 0.007 \\
\hline \multicolumn{6}{|l|}{ Categories } \\
\hline Q1 & 1429 (11.15\%) & ref & & ref & \\
\hline Q2-Q4 & $5313(13.74 \%)$ & $1.18(1.11-1.25)$ & $<0.001$ & $1.10(1.03-1.17)$ & 0.004 \\
\hline \multicolumn{6}{|l|}{ eGFR, $\mathrm{ml} / \mathrm{min} / 1.73 \mathrm{~m}^{2}$} \\
\hline Continuous variable per unit & & $0.984(0.983-0.985)$ & $<0.001$ & $0.990(0.989-0.992)$ & $<0.001$ \\
\hline \multicolumn{6}{|l|}{ Categories } \\
\hline$\geq 60$ & 4556 (10.95\%) & ref & & ref & \\
\hline$<60$ & $2186(22.12 \%)$ & $2.07(1.96-2.18)$ & $<0.001$ & $1.49(1.41-1.58)$ & $<0.001$ \\
\hline
\end{tabular}

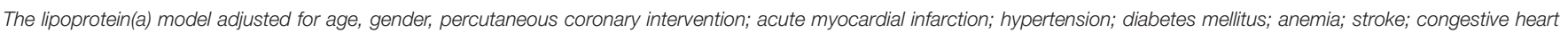

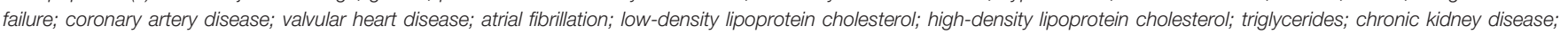
angiotensin-converting enzyme inhibitor or angiotensin receptor blocker; beta-blockers; statins.

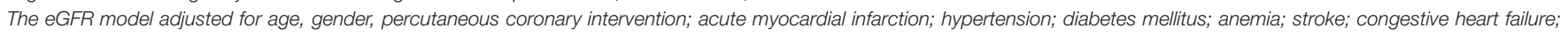

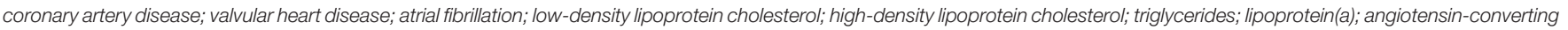
enzyme inhibitor or angiotensin receptor blocker; beta-blockers; statins.

or ARB among four groups (Q1, Q2, Q3, and Q4) (Table 1). Compared with patients with eGFR $\geq 60 \mathrm{~mL} / \mathrm{min} / 1.73 \mathrm{~m}^{2}$, patients with eGFR $<60 \mathrm{~mL} / \mathrm{min} / 1.73 \mathrm{~m}^{2}$ were older, and had a higher prevalence of HT, DM and lower LDL-C and HDLC (Table 1). Baseline characteristics across lipoprotein(a) and eGFR categories was shown in Supplementary Tables 1, 2. There were significant differences between the concentrations of lipoprotein(a) in different eGFR categories $(p<0.001)$ (Supplementary Figure 1).

\section{Lipoprotein(a), EGFR, and All-Cause Mortality}

During a median follow-up of 5.0 years (interquartile range: $3.0-7.6$ years), $13 \%(n=6,695)$ patients died. Compared with lipoprotein(a) Q1, lipoprotein(a) Q2, Q3, and Q4 were all associated with about $10 \%$ increased adjusted risk of death in all patients (Table 2). The cumulative hazard for all-cause mortality across lipoprotein(a) quartiles and eGFR categories shown in Supplementary Figure 2.

In patients with eGFR $<60 \mathrm{~mL} / \mathrm{min} / 1.73 \mathrm{~m}^{2}$, lipoprotein(a) Q2, Q3, and Q4 all had a 23\% increased adjusted risk of death relative to Q1 (All of $p<0.05$ ). In patients with eGFR $\geq 60 \mathrm{~mL} / \mathrm{min} / 1.73 \mathrm{~m}^{2}$, there were not significantly differences in adjusted risk of death between lipoprotein(a) Q2, Q3 as well as Q4 and Q1, respectively (Figure 1). In the subsequent analyses, lipoprotein(a) was dichotomized into low-risk group (Q1) and high-risk group (Q2-Q4). The cumulative hazard for all-cause mortality across lipoprotein(a) (Q1 vs. Q2-Q4) and eGFR categories shown in Figure 2. Compared with lipoprotein(a) Q1, lipoprotein(a) Q2-Q4 was strongly associated with increased adjusted risk of death in patients with eGFR $<60 \mathrm{~mL} / \mathrm{min} / 1.73 \mathrm{~m}^{2}$ (HR: 1.23 [95\% CI: 1.08-1.39]), while such association was not significant in patients with eGFR $\geq 60 \mathrm{~mL} / \mathrm{min} / 1.73 \mathrm{~m}^{2}$ (HR: 1.05 [95\% CI: 0.97-1.13]). P for interaction between lipoprotein(a) (Q1 vs. Q2-Q4) and eGFR ( $\geq 60$ vs. eGFR <60 $\mathrm{mL} / \mathrm{min} / 1.73 \mathrm{~m}^{2}$ ) on all-cause mortality was 0.019 (Table 3 ). More information on confounding variables of Table 3 can be detailed in Supplementary Table 3.

\section{DISCUSSIONS}

To our knowledge, this is the first large cohort real-world study to demonstrate the relationship between baseline eGFR and lipoprotein(a)-associated risk of all-cause mortality in patients undergoing CAG. Our main finding is that lipoprotein(a) has an eGFR- related modifier effect on all-cause mortality: higher lipoprotein(a) concentration has higher mortality in patients undergoing CAG with eGFR $<60 \mathrm{~mL} / \mathrm{min} / 1.73 \mathrm{~m}^{2}$, but this effect was not significant in patients $\mathrm{eGFR} \geq 60 \mathrm{~mL} / \mathrm{min} / 1.73 \mathrm{~m}^{2}$.

In the present study, elevated lipoprotein(a) level exhibited significant associations with the increased risk of all-cause mortality after correcting potentially confounding variables. In recent years, with the rapid development of medical technology and the rise of intervention of lipoprotein(a), baseline lipoprotein(a) has become the focus of research again. Many studies have proposed increased plasma lipoprotein(a) levels as an independent risk factor for long-term cardiovascular adverse events (18-21). In fact, lipoprotein(a) is an inherited 


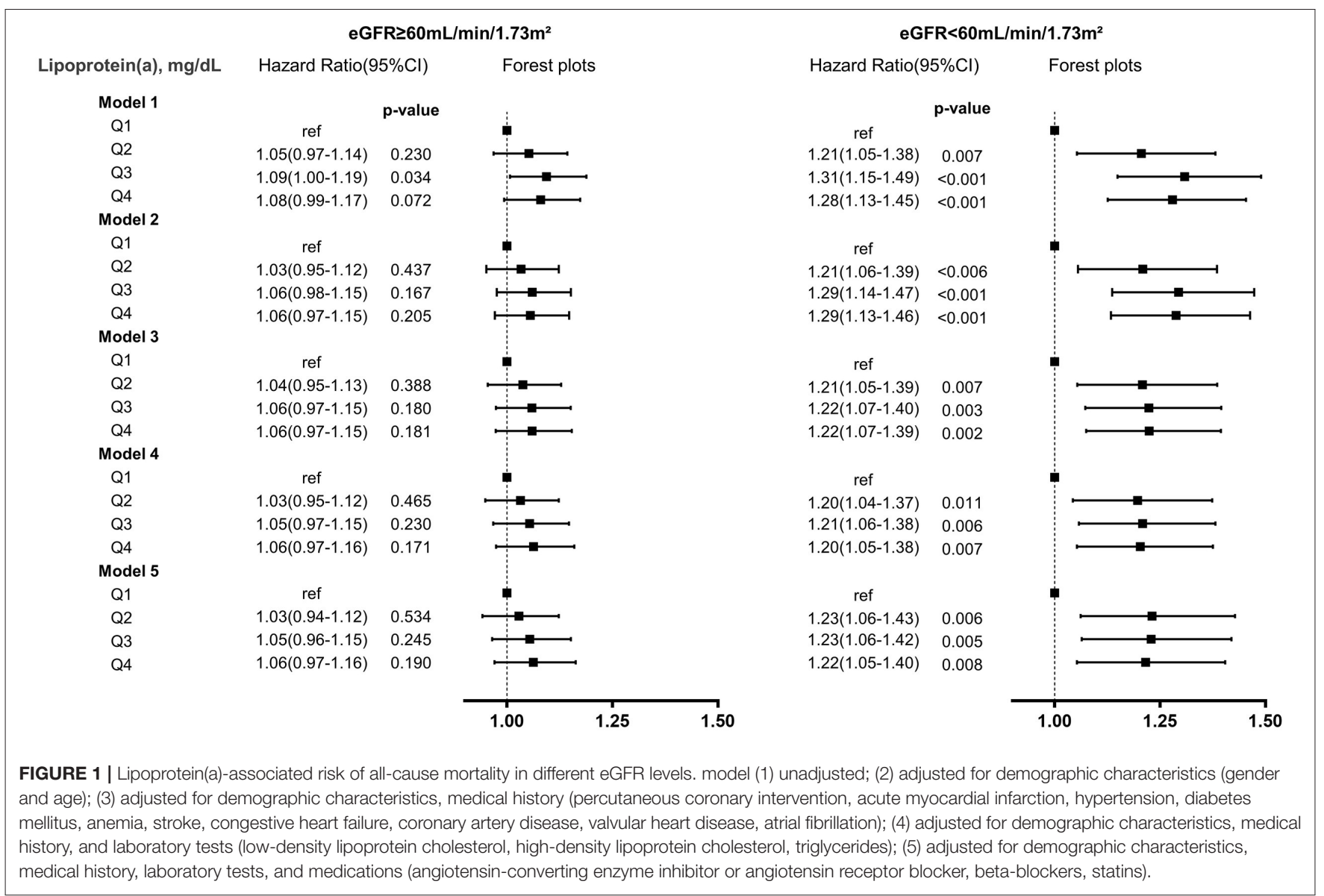

atherogenic lipoprotein, and more than $90 \%$ of the variance in concentrations can be explained by genetics (22). The synthesis and metabolism of lipoprotein(a) is independent with other lipoproteins or lipid components. Theoretically, there is no significant interaction between lipoprotein(a) and other lipoproteins or lipid. In addition, other lipoproteins containing LDL-C, HDL-C and TRIG have been recognized as associated with prognosis. Statins used to lower LDLC may increase plasma levels of the highly atherogenic molecule lipoprotein(a) (23). Considering the potential effects of lipoproteins and statins, our study has adjusted for those potential confounders. The possible underlying mechanism may be lipoprotein(a) potentially constitutes a molecular link between the processes of atherosclerosis (mediated by the LDL-like moiety) and thrombosis [mediated by the apo(a) moiety] that together precipitate events such as myocardial infarction and ischemic stroke $(24,25)$.

Renal function deteriorates, lipoprotein(a) concentrations increase. The association between kidney function and lipoprotein(a) levels has received interest since the initial observation of elevated lipoprotein(a) levels among dialysis patients in the end of 20th century (26). Previous studies showed the association of eGFR and lipoprotein(a) that higher lipoprotein(a) levels have been observed with reduced eGFR, even in the earliest stages of renal impairment
(24, 27-31). Our results also show that there is an inverse correlation between lipoprotein(a) and eGFR in patients undergoing CAG. The possible underlying mechanism may be that elevated plasmatic lipoprotein(a) levels in nephrotic patients could be linked to increased synthesis of proteins in the liver as physiological reaction to proteinuria $(3,12,23)$. The another potential hypotheses is that impaired renal function may lead to the decrease of the metabolic clearance rate, thus increasing the concentration of lipoprotein(a) (32).

It should be highlighted that a higher level of lipoprotein(a) in patients with poorer renal function tended to have a higher risk of all-cause mortality. In our study, the patients with relatively good renal function, elevated lipoprotein(a) had no significant risk of all-cause mortality, while the individuals with $\mathrm{eGFR}<60$, the higher level of lipoprotein(a) was associated with a sharp increased risk of all-cause mortality. Few studies have reported the association of eGFR and lipoprotein(a) and all-cause mortality. Recently, $\mathrm{Xu}$ et al. (18) found that high lipoprotein(a) value was associated with the occurrence of death in 427 consecutive patients who underwent PCI with CKD. Konishi et al. (19) also demonstrated that high lipoprotein(a) levels were associated with a composite of all-cause mortality and incident acute coronary syndrome in a cohort of 904 patients with CKD 


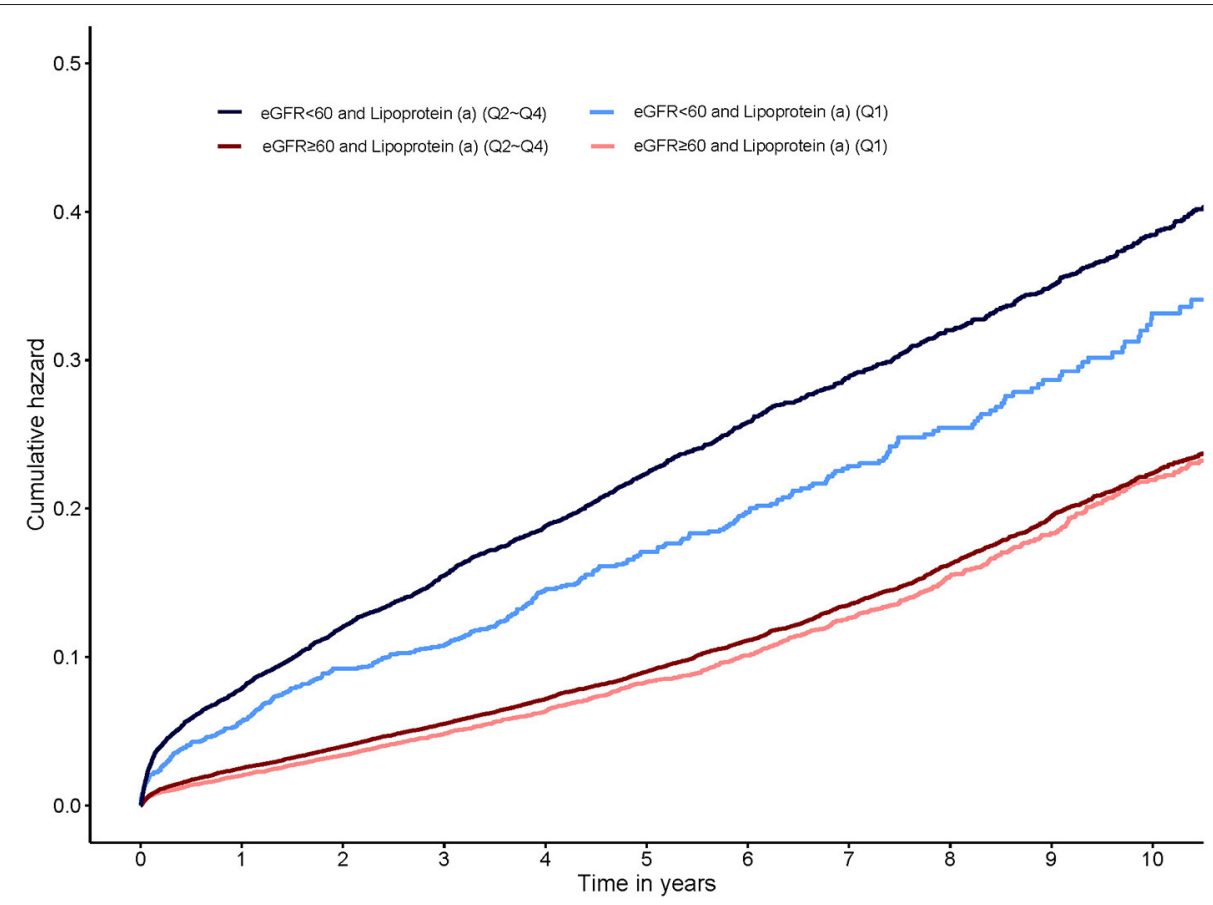

FIGURE 2 | Kaplan-Meier curves for cumulative hazard of all-cause mortality by lipoprotein(a) categories stratified eGFR.

TABLE 3 | HR of all-cause mortality across lipoprotein(a) categories in eGFR $\geq 60 \mathrm{~mL} / \mathrm{min} / 1.73 \mathrm{~m}^{2}$ and eGFR $<60 \mathrm{~mL} / \mathrm{min} / 1.73 \mathrm{~m}^{2}$.

\begin{tabular}{|c|c|c|c|c|c|}
\hline \multirow[t]{2}{*}{ Categories } & \multirow[b]{2}{*}{ Events (\%) } & \multicolumn{2}{|c|}{ Crude } & \multicolumn{2}{|c|}{ Adjusted } \\
\hline & & HR $(95 \% \mathrm{Cl})$ & $P$-value & HR (95\% Cl) & $P$-value \\
\hline \multicolumn{6}{|c|}{ eGFR $\geq 60 \mathrm{~mL} / \mathrm{min} / 1.73 \mathrm{~m}^{2}$} \\
\hline Lipoprotein(a)Q1 & 1074 (9.87\%) & ref & & ref & \\
\hline Lipoprotein(a)Q2-Q4 & 3482 (11.33\%) & 1.09 (1.01-1.16) & 0.019 & 1.05 (0.97-1.13) & 0.224 \\
\hline \multicolumn{6}{|c|}{ eGFR $<60 \mathrm{~mL} / \mathrm{min} / 1.73 \mathrm{~m}^{2}$} \\
\hline Lipoprotein(a)Q1 & 355 (18.35\%) & ref & & ref & \\
\hline Lipoprotein(a)Q2-Q4 & 1831 (23.04\%) & $2.29(2.13-2.47)$ & $<0.001$ & 1.23 (1.08-1.39) & 0.002 \\
\hline${ }^{*} \mathrm{P}$ for interaction & & $<0.001$ & & 0.019 & \\
\hline
\end{tabular}

${ }^{\star} p$-value for interaction test: 2-way interaction of lipoprotein(a) (Q1 vs. Q2-Q4) and renal function groups (eGFR $\geq 60 \mathrm{~mL} / \mathrm{min} / 1.73 \mathrm{~m}^{2}$ vs. eGFR $<60 \mathrm{~mL} / \mathrm{min} / 1.73 \mathrm{~m}^{2}$ ).

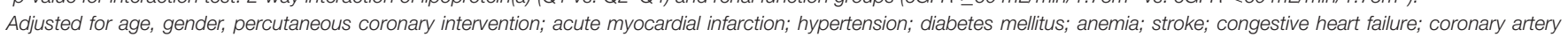

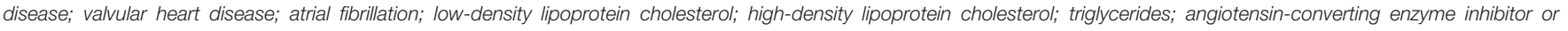
angiotensin receptor blocker; beta-blockers; statins.

among 3,508 patients who underwent the first PCI. However, studies on lipoprotein(a)-related risk of death focus on patients with poor renal function, the regulatory effect of eGFR stratification on lipoprotein(a)-associated mortality has not been adequately addressed. Our study showed that all-cause mortality is significantly increased in lipoprotein(a) (Q2-Q4) group compared with lipoprotein(a) Q1 in eGFR $<60 \mathrm{~mL} / \mathrm{min} / 1.73 \mathrm{~m}^{2}$, a difference not observed in eGFR $\geq 60 \mathrm{~mL} / \mathrm{min} / 1.73 \mathrm{~m}^{2}$. This finding may guide clinicians to develop the optimal individualized treatment strategy. The possible mechanism is that elevated lipoprotein (a) may contribute to the acceleration of glomerular injury in various renal diseases by inducing activation of reactive oxygen metabolites (33), and decreased renal catabolism may lead to accumulation of lipoprotein (a) and thus increased concentration (28), resulting in a vicious cycle of accelerated death.

All those findings strongly support the need for physicians to practice early risk stratification according to the level of lipoprotein(a) and eGFR in hospitalization. Clinicians should stay abreast of the current scientific evidence to provide the most meaningful and effective lipid metabolism control, aiming to individualize anti-atherosclerosis support. Although, lipoprotein(a) level is under strong genetic control and not susceptible to nutrition or physical activity $(22,34)$. Fortunately, several novel modalities of therapeutic interventions to lower plasma lipoprotein(a) concentrations 
have been shown. A clear effect of anti-atherosclerosis on lipoprotein(a)is exerted by protein convertase subtilisin/kexin type 9 (PCSK9) antagonists (35, 36). Currently, emerging therapies antisense oligonucleotides targeting apo(a) could potently reduce plasma lipoprotein(a) (37); However, these new drugs are too expensive to be universally available in a developing country. Meanwhile, reducing lipoprotein(a) concentration may not be that imperative in patients with $\mathrm{eGFR} \geq 60 \mathrm{~mL} / \mathrm{min} / 1.73 \mathrm{~m}^{2}$. It needs to be stressed to conduct clinical intensive management and the lipid-lowering therapy in patients with worse renal function and higher level of lipoprotein(a). Further studies are needed to prospectively evaluate the efficacy of lipoprotein(a) metabolism control on outcomes in patients with lower and higher levels of eGFR.

Limitations of this study should be considered. First, because it was a single-center, retrospective study, our inferences did not reflect direct causality. We must always recognize the potential for residual, uncontrolled confounding that might partly explain the associations. Secondly, there might be restrictions regarding generalization across ethnicities as we only included Chinese individuals; however, we are not aware of any data that the present results should not apply to people of most ethnicities. Thirdly, the sample size between lipoprotein(a) Q1 and lipoprotein(a) Q2-Q4 was indeed unequal. However, the large sample size can make up for this imbalance which may not affect the statistic validity. Forth, given that the eGFR and lipoprotein(a) concentration were conducted only at a single time point, we did not analyze the changes in lipoprotein(a) and renal status over time and their interaction with all-cause mortality. Fifth, there was limited data on the included patients, without information about proteinuria and albuminuria, which might help us to better understand the relationship between kidney function and lipoprotein(a). Last, although we established that eGFR might help classify risk in lipoprotein(a) categories, due to the observational nature of our study, prospective clinical trials are needed to establish whether aggressive lipoprotein(a) management in these groups will improve long-term outcomes.

\section{CONCLUSIONS}

Elevated lipoprotein(a) was associated with increased risk of all-cause mortality and such an association was modified by the baseline eGFR in CAG patients. More attention should be paid to the patients with reduced eGFR and elevated lipoprotein(a), and the appropriate lipoprotein(a) intervention is required. Future studies are needed to explore the potential mechanism of the association of

\section{REFERENCES}

1. Li C, Chen Q, Zhang M, Liu Y, Chu Y, Meng F, et al. The correlation between lipoprotein(a) and coronary atherosclerotic lesion is stronger than LDL-C, when LDL-C is less than $104 \mathrm{mg} / \mathrm{dL}$. lipoprotein(a) with all-cause mortality across categories of eGFR.

\section{DATA AVAILABILITY STATEMENT}

The original contributions presented in the study are included in the article/Supplementary Material, further inquiries can be directed to the corresponding authors.

\section{ETHICS STATEMENT}

All traceable personal identifiers were removed from the analytic dataset to protect patients' privacy. The study protocol was approved by Guangdong Provincial People's Hospital ethics committee and the study was performed according to the declaration of Helsinki.

\section{AUTHOR CONTRIBUTIONS}

ZDH, JLiu, SC, and LC: research idea and study design. ZDH, JLu, YYa, JLia, YH, YYu, HH, QL, BW, SL, ZY, DX, YL, KC, ZGH, and JN: data acquisition. JLiu and LC: data analysis/interpretation. $\mathrm{ZDH}$ and SL: statistical analysis. SC and LC: supervision and mentorship. LC: writing guidance. All authors contributed important intellectual content during manuscript drafting or revision and accepts accountability for the overall work by ensuring that questions on the accuracy or integrity of any portion of the work are appropriately investigated and resolved, and read and approved the final version.

\section{FUNDING}

This research was funded and supported by Fujian Province Natural Science Foundation (Grant number: 2019J01617), Beijing Lisheng Cardiovascular Health Foundation (No. LHJJ20141751), Study on the function and mechanism of the potential target for early warning of cardiorenal syndrome after acute myocardial infarction based on transformism (DFJH201919), Natural Science Foundation of Guangdong Province General Project (2020A1515010940), and Guangdong Provincial Key Laboratory of Coronary Heart Disease Prevention (2017B030314041). The funders had no role in the study design, data collection, and analysis, decision to publish, or preparation of the manuscript.

\section{SUPPLEMENTARY MATERIAL}

The Supplementary Material for this article can be found online at: https://www.frontiersin.org/articles/10.3389/fcvm. 2021.747120/full\#supplementary-material
BMC Cardiovasc Disord. (2021) 21:41. doi: 10.1186/s12872-02101861-6

2. Nordestgaard BG, Chapman MJ, Ray K, Borén J, Andreotti F, Watts GF, et al. Lipoprotein(a) as a cardiovascular risk factor: current status. Eur Heart J. (2010) 31:2844-53. doi: 10.1093/eurheartj/ehq386 
3. Kronenberg F, Utermann G. Lipoprotein(a): resurrected by genetics. J Intern Med. (2013) 273:6-30. doi: 10.1111/j.1365-2796.2012.02592.x

4. Tsimikas S, A. Test in context: lipoprotein(a): diagnosis, prognosis, controversies, and emerging therapies. J Am Coll Cardiol. (2017) 69:692711. doi: 10.1016/j.jacc.2016.11.042

5. Wu MF, Xu KZ, Guo YG Yu J, Wu Y, Lin LM. Lipoprotein(a) and atherosclerotic cardiovascular disease: current understanding and future perspectives. Cardiovasc Drugs Ther. (2019) 33:73948. doi: 10.1007/s10557-019-06906-9

6. Langsted A, Kamstrup PR, Nordestgaard BG. High lipoprotein(a) and high risk of mortality. Eur Heart J. (2019) 40:276070. doi: 10.1093/eurheartj/ehy902

7. Mach F, Baigent C, Catapano AL, Koskinas KC, Casula M, Badimon L, et al. 2019 ESC/EAS Guidelines for the management of dyslipidaemias: lipid modification to reduce cardiovascular risk. Eur Heart J. (2020) 41:11188. doi: 10.1093/eurheartj/ehz455

8. Grundy SM, Stone NJ, Bailey AL, Beam C, Birtcher KK, Blumenthal RS, et al. 2018 AHA/ACC/AACVPR/AAPA/ABC/ACPM/ADA/AGS/APhA/ASPC/NLA/ PCNA guideline on the management of blood cholesterol: executive summary: a report of the american college of cardiology/american heart association task force on clinical practice guidelines. Circulation. (2019) 139:e1046-81. doi: 10.1161/CIR.000000000 0000699

9. Tsimikas S, Viney NJ, Hughes SG, Singleton W, Graham MJ, Baker $\mathrm{BF}$, et al. Antisense therapy targeting apolipoprotein(a): a randomised, double-blind, placebo-controlled phase 1 study. Lancet. (2015) 386:147283. doi: 10.1016/S0140-6736(15)61252-1

10. O'Donoghue ML, Fazio S, Giugliano RP, Stroes ESG, Kanevsky E, Gouni-Berthold I, et al. Lipoprotein(a), PCSK9 inhibition, and cardiovascular risk. Circulation. (2019) 139:148392. doi: 10.1161/CIRCULATIONAHA.118.037184

11. Kronenberg F. Causes and consequences of lipoprotein(a) abnormalities in kidney disease. Clin Exp Nephrol. (2014) 18:234-7. doi: 10.1007/s10157-013-0875-8

12. Frischmann ME, Kronenberg F, Trenkwalder E, Schaefer JR, Schweer $\mathrm{H}$, Dieplinger $\mathrm{B}$, et al. In vivo turnover study demonstrates diminished clearance of lipoprotein(a) in hemodialysis patients. Kidney Int. (2007) 71:1036-43. doi: 10.1038/sj.ki. 5002131

13. K/DOQI clinical practice guidelines for chronic kidney disease: evaluation, classification, and stratification. Am J Kidney Dis. (2002)39:S1-266.

14. Manjunath G, Tighiouart H, Ibrahim H, MacLeod B, Salem DN, Griffith $\mathrm{JL}$, et al. Level of kidney function as a risk factor for atherosclerotic cardiovascular outcomes in the community. J Am Coll Cardiol. (2003) 41:4755. doi: 10.1016/S0735-1097(02)02663-3

15. Aguiar-Souto P, Ferrante G, Del Furia F, Barlis P, Khurana R, Di Mario C. Frequency and predictors of contrast-induced nephropathy after angioplasty for chronic total occlusions. Int J Cardiol. (2010) 139:6874. doi: 10.1016/j.ijcard.2008.10.006

16. Levey AS, Bosch JP, Lewis JB, Greene T, Rogers N, Roth D, et al. More accurate method to estimate glomerular filtration rate from serum creatinine: a new prediction equation. Modification of Diet in Renal Disease Study Group. Ann Intern Med. (1999) 130:461-70. doi: 10.7326/0003-4819-130-6-19990316000002

17. Mehran R, Aymong ED, Nikolsky E, Lasic Z, Iakovou I, Fahy M, et al. A simple risk score for prediction of contrast-induced nephropathy after percutaneous coronary intervention: development and initial validation. J Am Coll Cardiol. (2004) 44:1393-9. doi: 10.1016/S0735-1097(04) 01445-7

18. Xu N, Tang XF, Yao Y, Zhao XY, Chen J, Gao Z, et al. Association of plasma lipoprotein(a) with long-term adverse events in patients with chronic kidney disease who underwent percutaneous coronary intervention. Am J Cardiol. (2018) 122:2043-8. doi: 10.1016/j.jacc.2018. 08.559

19. Konishi H, Miyauchi K, Tsuboi S, Ogita M, Naito R, Dohi T, et al. Plasma lipoprotein(a) predicts major cardiovascular events in patients with chronic kidney disease who undergo percutaneous coronary intervention. Int $J$ Cardiol. (2016) 205:50-3. doi: 10.1016/j.ijcard.2015.12.007

20. Liu HH, Cao YX, Jin JL, Zhang HW, Hua Q, Li YF, et al. Predicting cardiovascular outcomes by baseline lipoprotein(a) concentrations: a large cohort and long-term follow-up study on real-world patients receiving percutaneous coronary intervention. J Am Heart Assoc. (2020) 9:e014581. doi: 10.1161/JAHA.119. 014581

21. Feng Z, Li HL, Bei WJ, Guo XS, Wang K, Yi SX, et al. Association of lipoprotein(a) with long-term mortality following coronary angiography or percutaneous coronary intervention. Clin Cardiol. (2017) 40:6748. doi: $10.1002 /$ clc. 22712

22. Jin JL, Cao YX, Zhang HW, Sun D, Hua Q, Li YF, et al. Lipoprotein(a) and cardiovascular outcomes in patients with coronary artery disease and prediabetes or diabetes. Diabetes Care. (2019) 42:1312-8. doi: 10.2337/dc19-0274

23. De Sain-Van Der Velden MG, Reijngoud DJ, Kaysen GA, Gadellaa MM, Voorbij H, Stellaard F, et al. Evidence for increased synthesis of lipoprotein(a) in the nephrotic syndrome. J Am Soc Nephrol. (1998) 9:147481. doi: 10.1681/ASN.V981474

24. Catena C, Colussi G, Nait F, Pezzutto F, Martinis F, Sechi LA. Early renal failure as a cardiovascular disease: focus on lipoprotein(a) and prothrombotic state. World J Nephrol. (2015) 4:374-8. doi: 10.5527/wjn. v4.i3.374

25. Boffa MB, Koschinsky ML. Lipoprotein (a): truly a direct prothrombotic factor in cardiovascular disease? J Lipid Res. (2016) 57:745-57. doi: 10.1194/jlr.R060582

26. Parra HJ, Mezdour H, Cachera C, Dracon M, Tacquet A, Fruchart JC. $L p(a)$ lipoprotein in patients with chronic renal failure treated by hemodialysis. Clin Chem. (1987) 33:721. doi: 10.1093/clinchem/ 33.5.721

27. Milionis HJ, Elisaf MS, Tselepis A, Bairaktari E, Karabina SA, Siamopoulos KC. apolipoprotein(a) phenotypes and lipoprotein(a) concentrations in patients with renal failure. Am J Kidney Dis. (1999) 33:1100-6. doi: 10.1016/S0272-6386(99)70147-2

28. Sechi LA, Zingaro L, De Carli S, Sechi G, Catena C, Falleti E, et al Increased serum lipoprotein(a) levels in patients with early renal failure. Ann Intern Med. (1998) 129:457-61. doi: 10.7326/0003-4819-129-6-19980915000006

29. Lin J, Reilly MP, Terembula K, Wilson FP. Plasma lipoprotein(a) levels are associated with mild renal impairment in type 2 diabetics independent of albuminuria. PLoS ONE. (2014) 9:e114397. doi: 10.1371/journal.pone. 0114397

30. Kovesdy CP, Astor BC, Longenecker JC, Coresh J. Association of kidney function with serum lipoprotein(a) level: the third National Health and Nutrition Examination Survey (1991-1994). Am J Kidney Dis. (2002) 40:899-908. doi: 10.1053/ajkd.2002. 36319

31. Kronenberg F, Kuen E, Ritz E, Junker R, König P, Kraatz G, et al. Lipoprotein(a) serum concentrations and apolipoprotein(a) phenotypes in mild and moderate renal failure. J Am Soc Nephrol. (2000) 11:105-15. doi: 10.1681/ASN. V111105

32. De Lima JJ. Increased serum lipoprotein(a) levels in patients with early renal failure. Ann Intern Med. (1999) 130:1028. doi: 10.7326/0003-4819-130-12-199906150-00013

33. Bronstein I, Voyta JC, Murphy OJ, Bresnick L, Kricka LJ. Improved chemiluminescent western blotting procedure. Biotechniques. (1992) 12:74853.

34. Mackinnon LT, Hubinger L, Lepre F. Effects of physical activity and diet on lipoprotein(a). Med Sci Sports Exerc. (1997) 29:1429-36. doi: 10.1097/00005768-19971100000007

35. Jawi MM, Frohlich J, Chan SY. Lipoprotein(a) the insurgent: a new insight into the structure, function, metabolism, pathogenicity, and medications affecting lipoprotein(a) molecule. J Lipids. (2020) 2020:3491764. doi: 10.1155/2020/3491764 
36. Greco MF, Sirtori CR, Corsini A, Ezhov M, Sampietro T, Ruscica M. Lipoprotein(a) lowering-from lipoprotein apheresis to antisense oligonucleotide approach. J Clin Med. (2020) 3:9. doi: 10.3390/jcm 9072103

37. Viney NJ, van Capelleveen JC, Geary RS, Xia S, Tami JA Yu $\mathrm{RZ}$, et al. Antisense oligonucleotides targeting apolipoprotein(a) in people with raised lipoprotein(a): two randomised, doubleblind, placebo-controlled, dose-ranging trials. Lancet. (2016) 388:2239-53. doi: 10.1016/S0140-6736(16)31009-1

Conflict of Interest: The authors declare that the research was conducted in the absence of any commercial or financial relationships that could be construed as a potential conflict of interest.
Publisher's Note: All claims expressed in this article are solely those of the authors and do not necessarily represent those of their affiliated organizations, or those of the publisher, the editors and the reviewers. Any product that may be evaluated in this article, or claim that may be made by its manufacturer, is not guaranteed or endorsed by the publisher.

Copyright (C) 2021 Huang, Yang, Lu, Liang, He, Yu, Huang, Li, Wang, Li, Yan, Xu, Liu, Chen, Huang, Ni, Liu, Chen and Chen. This is an open-access article distributed under the terms of the Creative Commons Attribution License (CC BY). The use, distribution or reproduction in other forums is permitted, provided the original author(s) and the copyright owner(s) are credited and that the original publication in this journal is cited, in accordance with accepted academic practice. No use, distribution or reproduction is permitted which does not comply with these terms. 